Biol. Proced. Online 2004;6(1): 173-179.

doi: $10.1251 /$ bpo 87

\title{
A novel method to analyze leukocyte rolling behavior in vivo
}

\author{
Jessica L. Dunne ${ }^{3}$, Adam P. Goobic ${ }^{2}$, Scott T. Acton² and Klaus Ley ${ }^{*}$
}

'Cardiovascular Research Center and Department of Biomedical Engineering, University of Virginia, PO Box 800759, Charlottesville, VA 22908. ${ }^{+}$Current address: Johnson \& Johnson Pharmaceutical R \& D, L.L.C., 1000 Route 202, Raritan, NJ 08869.

2Department of Electrical and Computer Engineering, University of Virginia, PO Box 400743, Charlottesville, VA 22904

${ }^{3}$ Cardiovascular Research Center and Department of Biomedical Engineering, University of Virginia, PO Box 800759, Charlottesville, VA 22908. Current address: Johnson \& Johnson Pharmaceutical R \& D, L.L.C., 1000 Route 202, Raritan, NJ 08869.

*To whom correspondence should be addressed: Phone: (434) 243-9966, Fax: (434) 924-2828, Email: klausley@virginia.edu

Submitted: May 20, 2004; Revised: August 4, 2004; Accepted: August 12, 2004; Published: August 27, 2004.

Indexing terms: Animals; Cell Adhesion; Leukocytes; Mice, Knockout; Tumor Necrosis Factor; Venules.

Abbreviations: ICAM-1, Intercellular Adhesion Molecule-1; LFA-1, Lymphocyte Function-Associated Antigen-1; Mac-1, Macrophage-1; TNF- $\alpha$, Tumor Necrosis Factor- $\alpha$.

\begin{abstract}
Leukocyte endothelial cell interaction is a fundamentally important process in many disease states. Current methods to analyze such interactions include the parallel-plate flow chamber and intravital microscopy. Here, we present an improvement of the traditional intravital microscopy that allows leukocyte-endothelial cell interaction to be studied from the time the leukocyte makes its initial contact with the endothelium until it adheres to or detaches from the endothelium. The leukocyte is tracked throughout the venular tree with the aid of a motorized stage and the rolling and adhesive behavior is measured off-line. Because this method can involve human error, methods to automate the tracking procedure have been developed. This novel tracking method allows for a more detailed examination of leukocyte-endothelial cell interactions.
\end{abstract}

\section{INTRODUCTION}

The accumulation of leukocytes in inflamed tissue results from adhesive interactions between leukocytes and endothelial cells within the microcirculation and requires several families of adhesion molecules. Adhesion molecules on leukocytes and endothelial cells interact with each other, initiating the adhesion cascade. Leukocytes proceed through capture, rolling, slow rolling, and adhesion before they transmigrate through the vascular endothelium to access interstitial tissue (1-10). Selectins, selectin ligands, integrins, and immunoglobulins are particularly important in the conversion of leukocytes from rolling to firm adhesion. Understanding the specific roles of each of these molecules may help in developing therapeutics against diseases with an inflammatory component, such as atherosclerosis, inflammatory bowel disease, or asthma.

Selectins are not only important for the initial capture and tethering of leukocytes to the endothelium, but also for leukocyte rolling. After treatment with $\mathrm{TNF}-\alpha$, E-selectin expression is induced on endothelial cells. E-selectin (E-/-) knockout mice have elevated leukocyte rolling velocities compared with wildtypes. E-selectin thus increases the transit time, which promotes firm adhesion in response to a local chemotactic stimulus $(3,11)$. In a study by Jung et al. (12), mice lacking L- and P-selectin (L-

(C) 2004 by the author(s). This paper is Open Access and is published in Biological Procedures Online under license from the author(s). Copying, printing, redistribution and storage permitted. Journal (C) 1997-2004 Biological Procedures Online. 
/P-), L- and E-selectin (L-/E-), or all three selectins (E-/L-/P-) reveal that any of the selectins can support some neutrophil recruitment, but eliminating all three selectins significantly impairs neutrophil recruitment. Similarly, in mice lacking $\beta_{2}$ integrins, leukocytes in TNF- $\alpha$-treated cremaster venules roll 3 times faster than in wild-type mice and fail to slow down and arrest on the endothelium $(2,5)$.

While rapid arrest of rolling leukocytes can be induced by high concentrations of chemoattractants (3) and soluble or surface bound chemoattractants have been implicated in the activation of leukocytes that transforms them into fully activated, adherent leukocytes (13-15), recent findings suggest that slow rolling via $\beta_{2}$ (CD18) integrins increases the transit time of leukocytes rolling through an inflamed vessel and may aid them in the transition from rolling to firmly adherent leukocytes $(2,3,16-18)$. This prolonged contact with the endothelium may be necessary to promote leukocyte activation and trigger integrin-mediated arrest and firm adhesion. While some leukocytes may adhere, or stick, to the endothelium after rolling along the vessel wall, it is also common for them to detach and return to free-flow $(5,16)$. Understanding how rolling leukocytes become adherent or detached is an important part of understanding the inflammatory cascade.

The process of neutrophil adhesion in vivo usually requires a rolling distance that exceeds a single microscopic field of view (200-300 $\mu \mathrm{m})$. Therefore, novel experimental assays to characterize cellular adhesion are needed to further the understanding of neutrophil-endothelial interactions during the inflammatory process. Current methods of analysis of these interactions are the parallel-plate flow chamber (19) and traditional intravital microscopy of the mouse cremaster muscle (20). However, parallel-plate flow chambers do not accurately mimic in vivo flow conditions. For one, the parallel-plate flow chamber uses isolated cells that may behave differently than they do in vivo. Furthermore, the flow chamber does not allow for pulsatile shear-flow conditions and realistic geometries similar to those encountered in small postcapillary venules, and most flow chamber assays do not include red blood cells. While traditional intravital microscopy represents actual physiological events, the analysis is incomplete in understanding the dynamic interactions between the leukocytes and the endothelium. Data is taken from a stationary frame, thus allowing only those interactions occurring within that field of view to be studied. By utilizing a moving field of view, one that follows a leukocyte from its initial contact with the endothelium to its eventual arrest or return to free flow, the means through which a leukocyte transitions from rolling to firmly arrested can begin to be explored. Utilizing this novel method, it has been shown that neutrophils systematically decelerate before adhering to the endothelium, while those that detach do not exhibit this behavior $(5,16)$, lending credence to the hypothesis that neutrophils integrate signals from the endothelial surface to reach a threshold of activation that allows them to adhere and eventually transmigrate.

\section{MATERIALS AND METHODS}

\section{Mice and reagents}

Breeding pairs of LFA-1 -/- (21) and Mac-1 -/- (22) mice were obtained from Dr. Christie M. Ballantyne (Department of Medicine, Baylor College of Medicine, Houston, TX). Breeding pairs of ICAM-1 null (23) mice were obtained from Dr. Arthur L. Beaudet (Department of Human Genetics, Baylor College of Medicine, Houston, TX). Control mice were age-matched C57BL/6 wild-type mice purchased from Hilltops Labs (Scottsdale, PA) or The Jackson Laboratory (Bar Harbor, ME). Only male mice were used because the cremaster muscle is investigated. Murine recombinant TNF- $\alpha(0.5 \mu \mathrm{g}$ per mouse $)$ was obtained from R\&D Systems (Minneapolis, MN).

\section{Preparation and surgery}

Two hours before exteriorization of the cremaster muscle, all mice were injected intrascrotally with $0.5 \mu \mathrm{g}$ TNF- $\alpha$ in $0.30 \mathrm{ml}$ isotonic saline. Mice were anesthetized with an intraperitoneal (i.p.) injection of a cocktail containing ketamine hydrochloride (Sanofi Winthrop Pharmaceuticals, $125 \mathrm{mg} / \mathrm{kg}$, New York, NY), xylazine (TranquiVed, Phoenix Scientific, Inc., $12.5 \mathrm{mg} / \mathrm{kg}$, St. Joseph, MO), and atropine sulfate (Fujisawa USA, Inc., 0.025 $\mathrm{mg} / \mathrm{kg}$, Deerfield, IL). In order to maintain body temperature, mice were laid on a heating pad that was kept at $37^{\circ} \mathrm{C}$. The trachea, jugular vein, and carotid arteries were cannulated with polyethylene (PE) tubing (Becton Dickinson and Company, Sparks, MD). The trachea cannula allowed for unobstructed breathing throughout the experiment. The jugular cannula was used to administer additional doses of anesthetics and saline for fluid balance as needed throughout the experiment. The carotid cannula was used to obtain systemic blood samples and inject mAbs.

\section{Intravital microscopy}

After surgery, the cremaster muscle was prepared for intravital microscopy as previously described $(20,24)$. Throughout exteriorization and the remainder of the experiment, the cremaster was superfused with a thermocontrolled $\left(37^{\circ} \mathrm{C}\right)$ bicarbonate-buffered saline $(131.9 \mathrm{mM} \mathrm{NaCl}, 18 \mathrm{mM} \mathrm{NaHCO} 3$, $4.7 \mathrm{mM} \mathrm{KCl}, 2.0 \mathrm{mM} \mathrm{CaCl} \bullet 2 \mathrm{H}_{2} 0$, and $1.2 \mathrm{mM} \mathrm{MgCl}_{2}$ ) equilibrated with $5 \% \mathrm{CO}_{2}$ in $\mathrm{N}_{2}$.

After exteriorization, the mouse was transported to the microscope stage. All microscopic observations were made on a Zeiss intravital microscope (Axioskop, Carl Zeiss, Inc., Thornwood, NY), with a saline immersion objective (SW 40/0.75 numerical aperture). Venules were videotaped through a CCD camera system (model VE-1000CD, Dage-MTI, Inc., Michigan City, IN) for approximately 90 seconds per venule on a VHS recorder (Panasonic AG-W1) for off-line analysis of leukocyte rolling velocity data. 


\section{Leukocyte tracking}

In order to better understand the cooperation between leukocyte rolling and adhesion, individual leukocytes were tracked through a venular tree upon initial contact with the endothelium at the confluence of two capillaries or the beginning of a postcapillary venule. After cremaster exteriorization, individual leukocytes, picked at random upon entering the tree from a capillary, were tracked using a motorized stage (McAllister Technical Services MC2000, Coeur d'Alene, ID) and intravital microscopy. Each leukocyte being tracked was followed until it adhered, detached from the endothelium, rolled out of the cremaster vasculature, or was lost due to technical limitations of the tracking procedure. Figure 1 shows a typical venular tree with diameter, length, velocity, and shear rate measurements. In larger vessels, the tracked leukocyte was often lost. Frequently, this was due to the tracked leukocyte rolling in an area congested with a large number of rolling or adherent leukocytes. The tracked leukocyte would often roll close to other rolling or adherent leukocytes, and it would become difficult or impossible to discern the original tracked leukocyte. However, the proportion of leukocytes that were lost was similar between groups (about $30 \%$, and thus, those leukocytes that were lost presumably did not affect the final analysis. An automatic tracking algorithm was developed to reduce human error. This method is based on active contours - flexible curves that can be used to automatically capture the cell boundary. Successful tracking requires vessel boundary detection (25), automated registration to reduce jitter (26), and computation of the cell boundaries in each using the active contours (27). The specific active contours used in this study are tailored to the size and shape of the leukocytes (27). More information about the automated tracker is available by contacting Dr. Scott T. Acton.

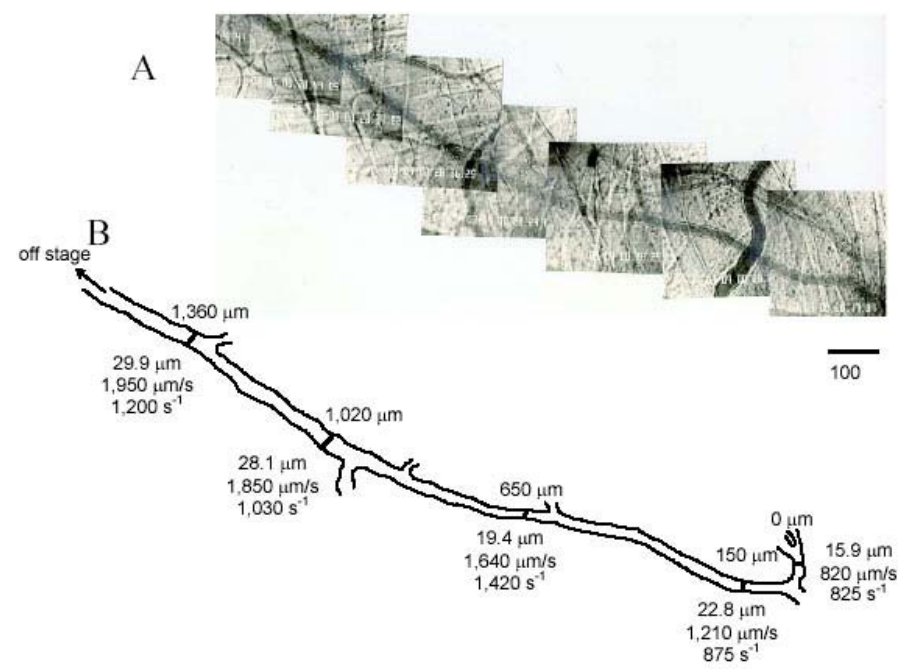

Fig. 1: Typical postcapillary venular tree in the mouse cremaster muscle. Photomicrograph montage of partial postcapillary venular tree in mouse cremaster muscle (A) with the corresponding tracing (B) showing important hemodynamic parameters including distance along tree (top) and venular diameter, centerline velocity, and calculated wall shear rate (bottom) (X5 objective; bar $=100$ $\mu \mathrm{m})$. Reproduced from Kunkel et al. (5), with permission. Copyright 2000 - The American Association of Immunologists, Inc.

\section{Adhesion and rolling velocity analysis}

Data obtained on videotapes during the experiments were analyzed off-line using a digital image processing system $(28,29)$. The position of each leukocyte was marked and the distance each leukocyte rolled in a constant 2-second window was measured. The rolling velocity was then calculated as $v_{r}=\frac{x_{r}}{2}$, where $\mathrm{x}_{\mathrm{r}}$ is the distance the leukocyte rolled during the 2-second time frame. A cell was defined as adherent when it was stationary for $>30$ seconds.

\section{Data and statistical analysis}

For each leukocyte studied, mean rolling velocity and average distance and time rolled were determined. The mean and SEM of rolling velocities was determined in the adherent and detached groups and statistical significance between groups was determined using a one-way ANOVA test at a significance level of $\mathrm{p}<0.05$. For improved comparison of data sets, cumulative velocity histograms were constructed by sorting all rolling velocities in ascending order and assigning each value $i$ a frequency equal to $i / n$ where $n$ is the total number of measurements in the group. The advantage of cumulative histograms is that two distributions can easily be compared in the same graph and that binning is not necessary. Cumulative histograms contain more information and less potential bias than traditional histograms.

For the leukocyte tracking studies, time and distance measurements were normalized to allow for superposition of various curves. Time and distance measurements were normalized by dividing their instantaneous value by the total time and distance each leukocyte traveled, respectively, as previously described (5). Each individual normalized curve was then averaged at fifty equidistant time points with the other leukocytes in its treatment group. This was performed separately for adherent and detached leukocytes within each treatment group. Distance versus time graphs were generated for each group. Figure 2 shows how two cumulative histograms are formed into one normalized cumulative histogram. The mean and SEM of rolling velocities was determined in the adherent and detached groups and statistical significance between groups was determined using a one-way ANOVA test at a significance level of $\mathrm{p}<0.05$. The percentage, or proportion of leukocytes that adhered for each group was also studied. In order to determine statistical significance between groups, tests on the proportions of adherent leukocytes were performed at a significance level of $\mathrm{p}<0.05$ using a $Z$ statistic. 

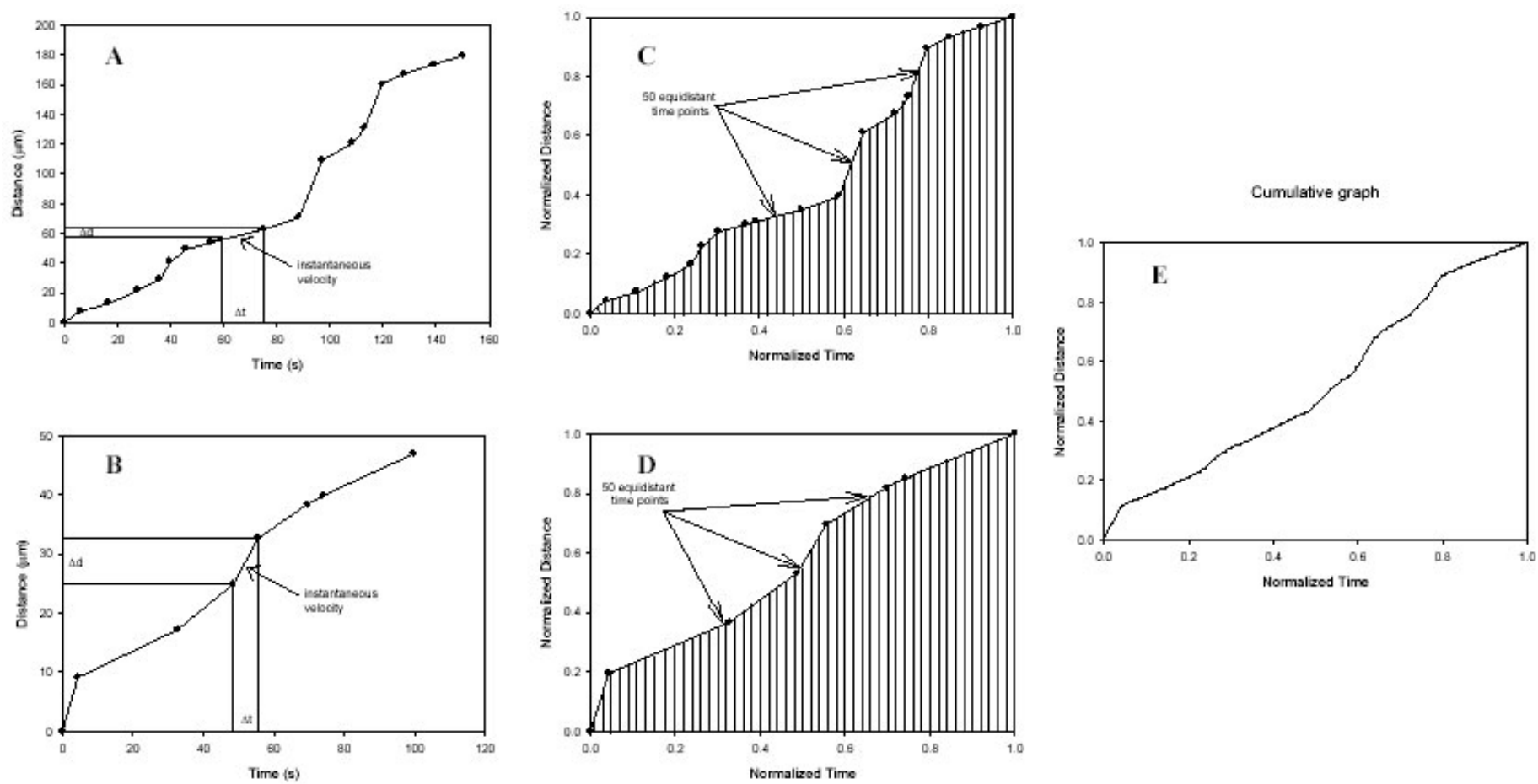

Fig. 2: The progression of individual cumulative histograms into a single, normalized cumulative histogram. Individual histograms are plotted in (A) and (B). Instantaneous velocity can be determined from the slope of the line between two distance-time points. (C) and (D) show the plots in (A) and (B), respectively, normalized and binned into fifty equidistant time points. The corresponding distance point for each time point is extrapolated by assuming a linear velocity between adjacent distance-time points. (E) shows the resulting merge of (C) and (D).

\section{RESULTS}

\section{Roles of LFA-1, Mac-1, and ICAM-1 in leukocyte arrest during cytokine-induced inflammation}

Intravital microscopy has been used extensively to study the rolling and adhesive behavior of leukocytes in vivo $(20,24)$. The process by which rolling leukocytes adhere to the endothelium is thought to be mediated by $\beta_{2}$ integrins following activation. To test this hypothesis, individual leukocytes were tracked down a venular tree in a TNF- $\alpha$ treated cremaster until they adhered, detached from the endothelium, or were lost due to technical limitations. Leukocytes were tracked down the vasculature of wild-type mice, LFA-1 -/- mice, Mac-1 -/-, and ICAM-1 ${ }^{\text {null }}$ mice to determine the individual roles of LFA-1, Mac-1, and ICAM-1 in the conversion of a rolling leukocyte to a firmly adherent one.

Twenty-one of twenty-two leukocytes tracked in the wild-type mice adhered to the endothelium (Fig. 3). In LFA-1 -/- mice, only nine out of twenty-nine leukocytes adhered, and in Mac-1 /- mice, eleven out of twenty-one tracked leukocytes adhered. Nine out of twenty-seven leukocytes $(33 \%)$ adhered to the endothelium in ICAM-1 ${ }^{\text {null }}$ mice. These data suggest that LFA-1, Mac-1, and ICAM- $1^{\mathrm{n}}$ are involved in the adhesion of leukocytes to the inflamed endothelium.
$N=22 \quad 27 \quad 21$

29

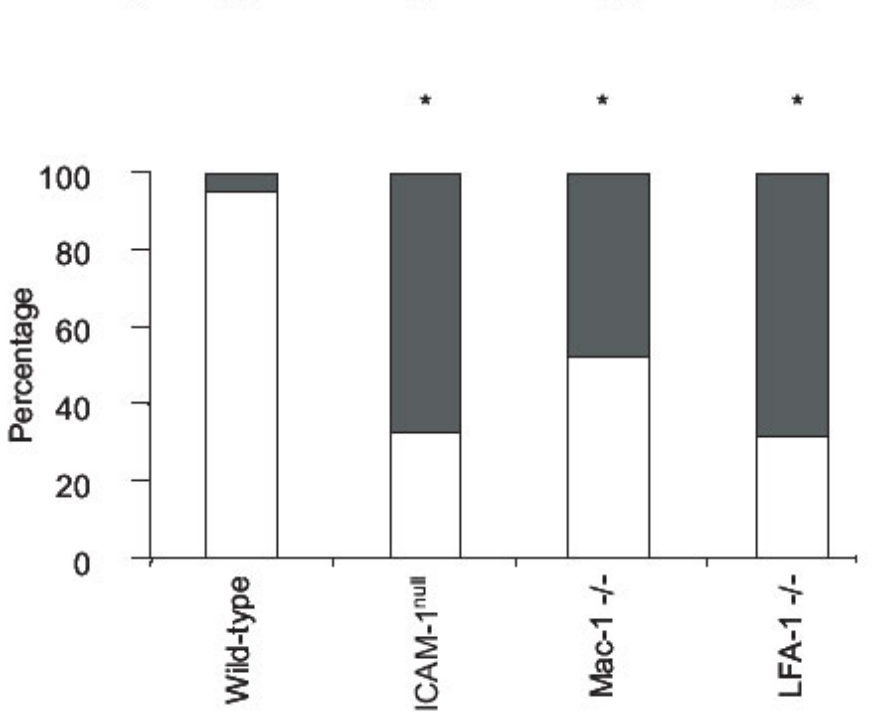

Fig. 3: Percentage of leukocytes that adhered or detached. $\square$, the percentage of leukocytes that adhered. $\mathbf{m}$, the percentage of leukocytes that detached. The number of leukocytes tracked per mouse treatment is indicated above the bars. *, those percentages of adherent leukocytes that are significantly lower than wild type. Modified from Dunne et al. (16), reproduced with permission. Copyright 2003 - The American Association of Immunologists, Inc. 
To examine the behavior of rolling leukocytes as they adhere or detach from the endothelium, cumulative distance versus time plots were created for each group. To compare each group on the same graph, rolling distance and time were each normalized by dividing the instantaneous value by the total time and distance for each leukocyte, respectively $(5,16)$. Each individual normalized curve was then averaged at fifty equidistant time points with the curves corresponding to the other leukocytes in the same treatment group. This was performed separately for adherent and detached leukocytes within each treatment group. Distance versus time graphs were generated for each group (Fig. 4). Leukocytes that adhered had distance versus time curves that showed decelerating behavior, while those leukocytes that detached exhibited accelerating or non-decelerating distance versus time curves. The shape of curves representing leukocytes that eventually adhere suggests gradual deceleration and thus progressive activation of rolling leukocytes.

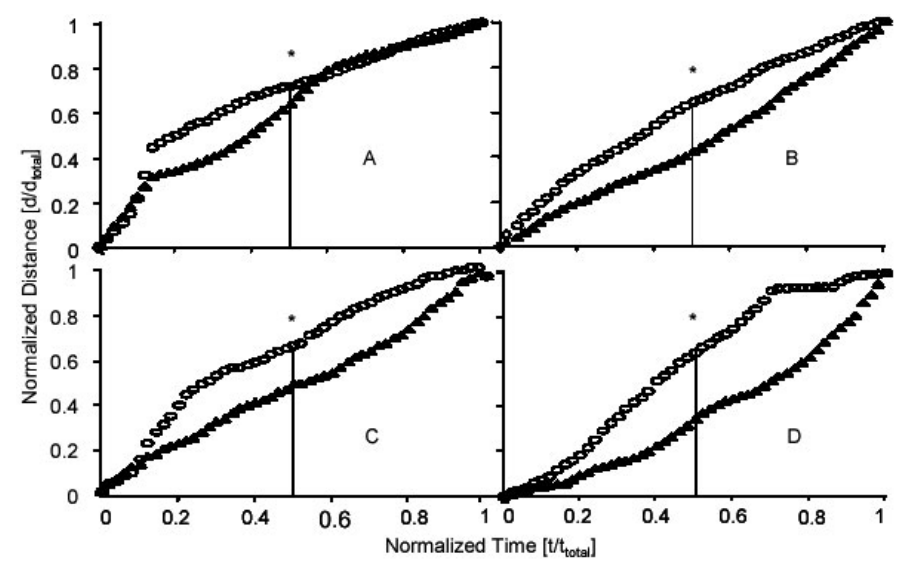

Fig. 4: Normalized distance vs. normalized time curves. Wild-type (A), ICAM-1 ${ }^{\text {null }}(B)$, Mac-1 -/- (C), and LFA-1 -/- (D). $\circ$, the tracings of those leukocytes that eventually adhered to the endothelium. $\boldsymbol{\Delta}$, the tracings of those leukocytes that eventually detached from the endothelium. *, significant difference between median values (vertical line). Modified from Dunne et al. (16), reproduced with permission. Copyright 2003 - The American Association of Immunologists, Inc.

To investigate whether duration of contact was important in the eventual fate of an individual leukocyte, the total distance and time traveled by each leukocyte was recorded. Wild-type leukocytes that adhered rolled for an average of $272 \pm 58 \mu \mathrm{m}$ before adhering, while the one wild-type leukocyte that detached rolled significantly longer $(411 \mu \mathrm{m})$ before detaching. Similarly, leukocytes from Mac-1 -/- and LFA-1 -/- mice that eventually detached rolled for longer distances than those that adhered $(1285 \pm 179 \mu \mathrm{m}$ vs. $386 \pm 113 \mu \mathrm{m}$ and $916 \pm 126 \mu \mathrm{m}$ vs. $565 \pm$ $170 \mu \mathrm{m}$, respectively). While the one wild-type leukocyte that detached rolled for a significantly longer time (164 seconds) than those wild-type leukocytes that adhered $(86 \pm 18$ seconds), leukocytes in Mac-1 -/- and LFA-1 -/- mice rolled for similar times, regardless of whether they adhered or detached $(285 \pm 79$ seconds vs. $423 \pm 56$ seconds and $218 \pm 71$ seconds vs. $169 \pm 46$ seconds, respectively) (Fig. 5).
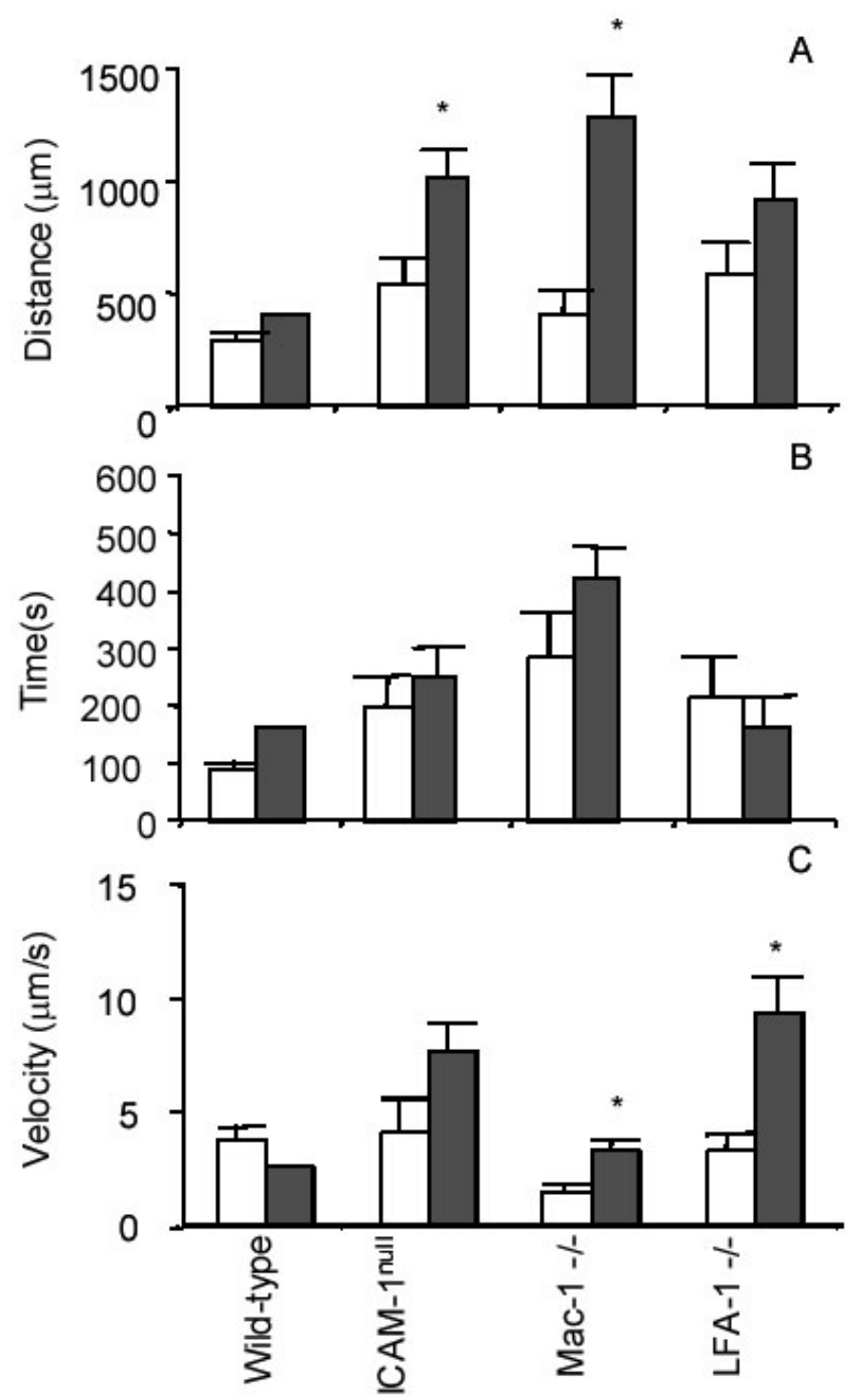

Fig. 5: Average distance rolled, time rolled, and average rolling velocity. Distance rolled (A), time rolled (B), and rolling velocity (C). Rolling velocities are based on the total distance and time a leukocyte is rolling along the endothelium until it adheres or detaches. Because leukocytes often pause along the endothelium as they are rolling, these pauses are counted in the total time. $\square$, the averages of those leukocytes that eventually adhered. - the averages of those leukocytes that eventually detached. Data are presented as mean \pm SEM. $*$, those values in detached leukocytes that are significantly different from the values in adherent leukocytes. Modified from Dunne et al. (16), reproduced with permission. Copyright 2003 - The American Association of Immunologists, Inc.

\section{DISCUSSION AND FUTURE DIRECTIONS}

In this report, we describe methods to study the molecular mechanisms of rolling and arrest of individual leukocytes in vivo during the early stages of the inflammatory process. We describe a novel method of tracking individual leukocytes in lieu of the more traditional rolling velocity analysis using intravital microscopy. While this new method can be rather labor intensive, the resulting data is more informative than traditional rolling velocity analysis. Here, we have shown that leukocytes decelerate before adhering to the endothelium, while those that eventually 
detach from the endothelium do not exhibit progressive deceleration. However, those leukocytes that detach are in contact with the endothelium for similar periods of time and distance as their counterparts that adhere, suggesting that these leukocytes are not integrating signal efficiently.
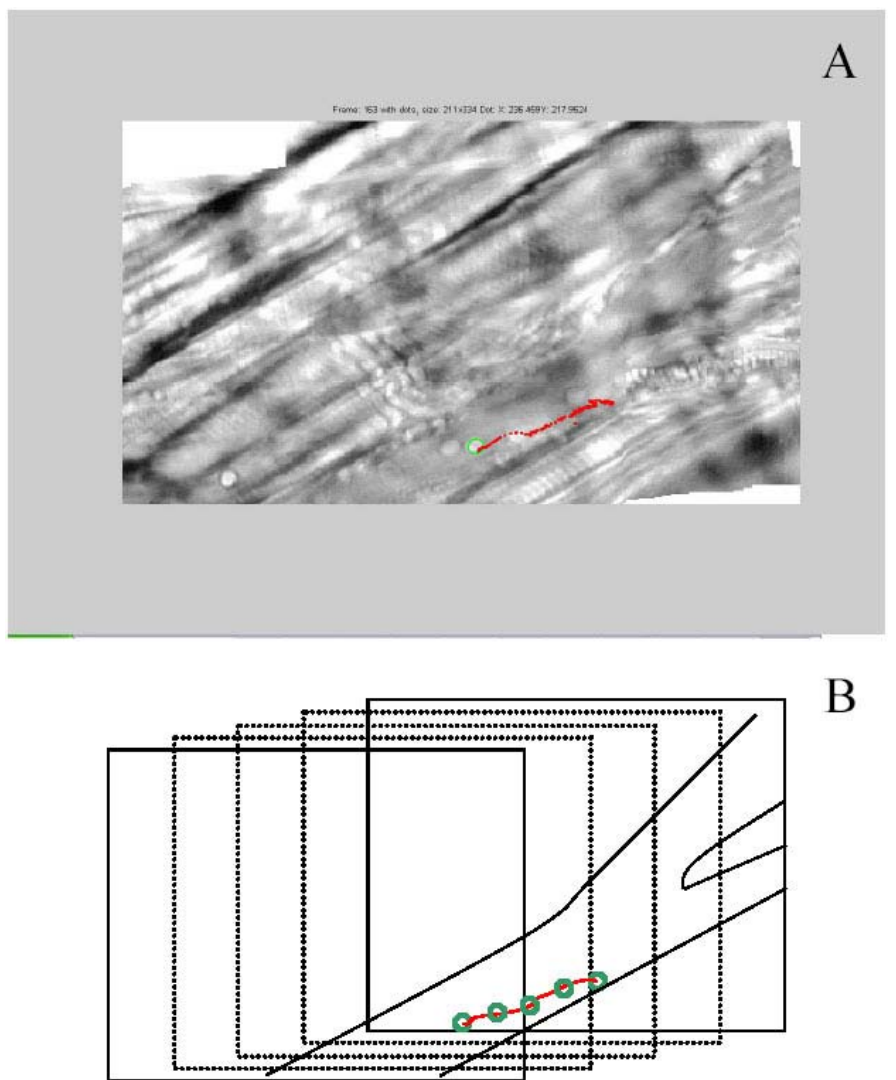

B

Fig. 6: (A) Path traveled by tracked leukocyte during transition. Tracked leukocyte indicated by green circle. Path the leukocyte traveled indicated in red. (B) schematic of path in (A), showing three transition slides in the path and the location of the leukocyte as indicated by the green circles.

While beneficial to the understanding of leukocyte behavior, tracking leukocytes has its disadvantages as well. In addition to being labor intensive, leukocytes are often lost due to the technical limitations of the tracking procedure. However, research is currently underway to automate the leukocyte tracking system, which could possibly eliminate these potential drawbacks to this powerful research tool. The ability to automatically track leukocytes in an in vivo setting will allow for more accurate determination of rolling distance and thus velocity of leukocytes in the cremaster vasculature. Here, the intravital microscopy video is stored as audio-video interleaved (AVI) files. An active contour (snake) tracker was used to automatically track leukocyte positions in each frame. Another advantage of the automated approach is the ability to register the frames in space, thus reducing the position error due to movement of the background. In the live mouse model, the recorded cremaster image is in constant motion, mostly due to the respiratory movements of the mouse. The respiratory movements can be accounted for to a degree in manual tracking. That is, measurements can be taken at the same point in the respiratory cycle, for example, at the end of expiration. However, this does not perfectly eliminate the motion, or jitter, associated with respiratory movements. On the other hand, because the automatic tracker first registers the background, movement associated with a live mouse is eliminated. Briefly, prior to tracking, a correlation-based stabilization algorithm is applied to minimize the constant global motion, or jitter, caused by the respiratory system. The algorithm uses morphological techniques to remove the venule from each displaced frame. Patches are then selected and matched to corresponding patches in a reference frame to determine the corrective translation. Automated tracking in a moving field of view is achieved with the assistance of a correlation-based registration algorithm which essentially determines the amount of translation experience by the moving stage. Figure 6 shows an example image mosaic created using the registration algorithm. Our experiments show that the automated registration removes an average (average of root mean squared errors) of $3.10 \mu \mathrm{m}$ in registration error per frame (using 15 sequences of 31 frames each). While this automated tracker is an improvement over the manual tracker in terms of analysis time and accuracy, the current limitations of the tracker hinder its implementation.

In conclusion, the novel tracking method reported here indicates the long endothelial contact time and gradual deceleration required for most leukocytes to transition from slowly rolling leukocytes to firmly adherent ones on the inflamed endothelium. Prior to this technology, such analysis was not possible. Contrary to prior reports in flow chambers that indicated that leukocyte arrest was rapid, (13-15) leukocytes in vivo to undergo a progressive activation suggested by their deceleration and a graded calcium flux (5). Using this analysis, future studies will be able to look at the effects of integrin antagonists and chemotactic factors on the adhesion process.

\section{ACKNOWLEDGMENTS}

This work was supported by NIH HL 54136 to K.L. and 68510 to S.A.

\section{REFERENCES}

1. Butcher EC. Leukocyte-endothelial cell recognition: three (or more) steps to specificity and diversity. Cell 1991; 67(6):10331036.

2. Jung U, Norman KE, Scharffetter-Kochanek K, Beaudet AL, Ley K. Transit time of leukocytes rolling through venules controls cytokine-induced inflammatory cell recruitment in vivo. J Clin Invest 1998; 102(8):1526-1533.

3. Ley K, Allietta M, Bullard DC, Morgan S. Importance of Eselectin for firm leukocyte adhesion in vivo. Circ Res 1998; 83(3):287-294.

4. Milstone DS, Fukumura D, Padgett RC, O'Donnell PE, Davis VM, Benavidez OJ et al. Mice lacking E-selectin show normal numbers of rolling leukocytes but reduced leukocyte stable arrest on cytokine-activated microvascular endothelium. Microcirculation 1998; 5(2-3):153-171. 
5. Kunkel EJ, Dunne JL, Ley K. Leukocyte arrest during cytokine-dependent inflammation in vivo. I Immunol 2000; 164(6):3301-3308.

6. Forlow SB, White EJ, Barlow SC, Feldman SH, Lu H, Bagby GJ et al. Severe inflammatory defect and reduced viability in CD18 and E-selectin double-mutant mice. J Clin Invest 2000; 106(12):1457-1466.

7. Stein JV, Cheng G, Stockton BM, Fors BP, Butcher EC, von Andrian UH. L-selectin-mediated leukocyte adhesion in vivo: microvillous distribution determines tethering efficiency, but not rolling velocity. J Exp Med 1999; 189(1):37-50.

8. Xu H, Manivannan A, Goatman KA, Jiang HR, Liversidge J, Sharp PF et al. Reduction in shear stress, activation of the endothelium, and leukocyte priming are all required for leukocyte passage across the blood-retina barrier. I Lenkoc Biol 2004; 75(2):224-232.

9. Sikora L, Johansson AC, Rao SP, Hughes GK, Broide DH, Sriramarao P. A murine model to study leukocyte rolling and intravascular trafficking in lung microvessels. Am J Pathol 2003; 162(6):2019-2028.

10. Grayson MH, Hotchkiss RS, Karl IE, Holtzman MJ, Chaplin DD. Intravital microscopy comparing $\mathrm{T}$ lymphocyte trafficking to the spleen and the mesenteric lymph node. $\mathrm{Am}$ J Physiol Heart Circ Physiol 2003; 284(6):H2213-H2226.

11. Kunkel EJ, Ley K. Distinct phenotype of E-selectindeficient mice. E-selectin is required for slow leukocyte rolling in vivo. Circ Res 1996; 79(6):1196-1204.

12. Jung U, Ley $K$. Mice lacking two or all three selectins demonstrate overlapping and distinct functions for each selectin. J Immunol 1999; 162(11):6755-6762.

13. Campbell JJ, Hedrick J, Zlotnik A, Siani MA, Thompson DA, Butcher EC. Chemokines and the arrest of lymphocytes rolling under flow conditions. Science 1998; 279(5349):381384.

14. Rainger GE, Fisher AC, Nash GB. Endothelial-borne platelet-activating factor and interleukin-8 rapidly immobilize rolling neutrophils. Am J Physiol 1997; 272(1 Pt 2):H114H122.

15. Gerszten RE, Garcia-Zepeda EA, Lim YC, Yoshida M, Ding HA, Gimbrone MA, Jr. et al. MCP-1 and IL-8 trigger firm adhesion of monocytes to vascular endothelium under flow conditions. Nature 1999; 398(6729):718-723.

16. Dunne JL, Collins RG, Beaudet AL, Ballantyne CM, Ley K. Mac-1, but Not LFA-1, Uses Intercellular Adhesion Molecule-1 to Mediate Slow Leukocyte Rolling in TNFalpha-Induced Inflammation. I Immunol 2003; 171(11):61056111.

17. Dunne JL, Ballantyne CM, Beaudet AL, Ley K. Control of leukocyte rolling velocity in TNF-alpha-induced inflammation by LFA-1 and Mac-1. Blood 2002; 99(1):336341.

18. Simon SI, Hu Y, Vestweber D, Smith CW. Neutrophil tethering on E-selectin activates beta 2 integrin binding to ICAM-1 through a mitogen-activated protein kinase signal transduction pathway. J Immunol 2000; 164(8):4348-4358.

19. Forrester JV, Lackie JM. Adhesion of neutrophil leucocytes under conditions of flow. J Cell Sci 1984; 70:93-110.
20. Ley K, Bullard DC, Arbones ML, Bosse R, Vestweber D, Tedder TF et al. Sequential contribution of L- and P-selectin to leukocyte rolling in vivo. J Exp Med 1995; 181(2):669-675.

21. Ding ZM, Babensee JE, Simon SI, Lu H, Perrard JL, Bullard DC et al. Relative contribution of LFA-1 and Mac-1 to neutrophil adhesion and migration. J Immunol 1999; 163(9):5029-5038.

22. Lu H, Smith CW, Perrard J, Bullard D, Tang L, Shappell SB et al. LFA-1 is sufficient in mediating neutrophil emigration in Mac-1-deficient mice. J Clin Invest 1997; 99(6):1340-1350.

23. Collins RG, Richer SE, Robker RL, Smith CW, Beaudet AL. ICAM-1 null mice differ from previous ICAM-1 mutants: Is your model affected? Keystone Symposia. 2003. Ref Type: Abstract.

24. Baez S. An open cremaster muscle preparation for the study of blood vessels by in vivo microscopy. Microvasc Res 1973; 5(3):384-394.

25. Tang J, Acton ST. Vessel boundary tracking for intravital microscopy via multiscale gradient vector flow snakes. IEEE Trans Biomed Eng 2004; 51(2):316-324.

26. Goobic AP, Tang J, Acton ST. Image stabilization and registration for tracking cells in the microvasculature. IEEE Trans Biomed Eng. In press.

27. Ray N, Acton ST, Ley K. Tracking leukocytes in vivo with shape and size constrained active contours. IEEE Trans Med Imaging 2002; 21(10):1222-1235.

28. Acton ST, Wethmar K, Ley K. Automatic tracking of rolling leukocytes in vivo. Microvasc Res 2002; 63(1):139-148.

29. Norman KE. An effective and economical solution for digitizing and analyzing video recordings of the microcirculation. Microcirculation 2001; 8(4):243-249. 